\title{
Cascade position- torque control strategy based on function approximation technique (FAT) for flexible joint robots
}

Hayder F.N. Al-Shuka ( $\nabla$ dr.hayder.f.n@coeng.uobaghdad.edu.iq )

https://orcid.org/0000-0002-4041-385X

\section{Burkhard Corves}

Ehab N. Abbas

\section{Research Article}

Keywords: Flexible joint, adaptive approximation control, decentralized control, torque control

Posted Date: December 30th, 2021

DOl: https://doi.org/10.21203/rs.3.rs-1197021/v2

License: (c) (i) This work is licensed under a Creative Commons Attribution 4.0 International License.

Read Full License 


\title{
Cascade position- torque control strategy based on function approximation technique (FAT) for flexible joint robots
}

\author{
Hayder F.N. Al-Shuka ${ }^{1, a)}$, Burkhard Corves ${ }^{2}$ ) , and Ehab N. Abbas ${ }^{3, c)}$ \\ ${ }^{1}$ Department of Aeronautical Engineering, College of Engineering, University of Baghdad, Baghdad, Iraq \\ ${ }^{2}$ Institute of Mechanism Theory, Machine Dynamics and Robotics, RWTH University, Aachen, Germany \\ ${ }^{3}$ Ministry of Higher Education and Scientific Research, Studies and Planning and Follow Up Directorate, Baghdad, \\ Iraq \\ a)dr.hayder.f.n@coeng.uobaghdad.edu.iq \\ b) corves@igmr.rwth-aachen.de \\ c) ehab19722002@gmail.com \\ a) Corresponding author: dr.hayder.f.n@coeng.uobaghdad.edu.iq
}

\begin{abstract}
This work deals with control of rigid link robotic manipulators provided with flexible joints. Due to presence of flexible joint dynamics, additional degrees of freedom and underactuation are developed that would complicate the control design. Besides, model uncertainties, unmodeled dynamics and disturbances should be considered in robot modeling and control. Therefore, this paper proposes a cascade position-torque control strategy based on function approximation technique (FAT). The key idea is to design two nested loops: 1) an outer position control loop for tracking reference trajectory, and 2) an inner joint torque control loop to track the desired joint torque resulted from the outer position loop. The torque control loop makes the robot system more adaptable and compliant for sudden disturbances. It increases the perception capability for the target robot mechanisms. Adaptive approximation control (AAC) is used as a strong tool for dealing with time-varying uncertain parameters and disturbances. A sliding mode term is easily integrated with control law structure; however, a constraint on feedback gains are established for compensating modeling (approximation) error. The proposed control architecture can be easily used for high degrees of freedom robotic system due to the decentralized behavior of the AAC. A two-link manipulator is used for simulation experiments. The simulated robot is commanded to move from rest to desired step references considering three cases depending on the selected value of the sliding mode time constant. It is shown that selection of a large time constant parameter related to the position loop leads to slow response. Besides, one of the inherent issues associated with the inner torque control is the presence of derivative of desired joint torque that makes the input control abruptly jumping at the beginning of the dynamic response. To end this, an approximation for derivative term of the desired joint torque is established using a low-pass filter with a time constant selected carefully such that a feasible dynamic response is ensured.The results show the effectiveness of the proposed controller.
\end{abstract}

Keywords. Flexible joint; adaptive approximation control, decentralized control, torque control

\section{INTRODUCTION}

Robotic manipulators usually have transmission elements with some flexibility, e.g. harmonic drives have considerable joint flexibility [1]. Adding flexible elements to transmission drives could lead to low output impedance, increase robustness when working in unstructured environments and enlarge the output torque [2]. Incorporation of flexible elements in joint design makes difficulties in modeling and control due to the following [3]:

- An additional degree of freedom is obtained since the motor and link angles are no longer equivalent.

- The order of the flexible dynamic system becomes twice the rigid joint. 
- The related dynamics undergo underactuation behaviour since the number of control input is less than the order of the target system.

- The existence of flexible elements causes fast dynamics that complicate modeling and control issues.

- Uncertainty in drive and link dynamics complicates the control design.

Thus, the flexible joint has more complex modeling than a rigid joint. Miscellaneous control approaches have been reported in the literature for motion control of flexible-joint robots. Feedback linearization control has been used extensively that achieves linearized closed-loop dynamics with guaranteed global asymptotic stability. However, these controllers may require measurements of high-order derivatives for the output variables (robot link positions) which is undesirable [4-6]. Backstepping control has similar features to the feedback linearization technique [7]. On the other hand, singular perturbation control is a powerful tool for controlling joints with high stiffness; however, it is impractical for moderate and low stiffness joints [8]. Furthermore, other control techniques are possible such as passivity-based control [9], adaptive control [10], and PID family control [11]; however, these controllers usually focus on position control loop with less attention on joint torque loop [4]. The torque control loop makes the robot system more adaptable and compliant for sudden disturbances. Therefore, Li et al. [12] have proposed a position/torque switching control strategy to improve robot safety for human-robot applications. The key idea is that during a collision with sudden disturbances, torque control is activated such that the impact force is attenuated or scaled within a safe range. In effect, the dynamics of the flexible-joint robotic manipulator can be decomposed into two subsystems, link and joint subsystems. For the link subsystem, the output state variable vector is the angular link position vector and the virtual input control is the interaction spring torque (or so-called the joint torque). Whereas for the joint (actuator) subsystem, the output state variable vector is the joint torque and the input is the motor voltage. Thus, a two-stage control can be designed to get feasible performance. The two-stage loops include coupled linkactuator dynamics with possible uncertainty such as the link-joint parameters, friction models, spring and geartransmission nonlinearity etc. Consequently, adaptive and or robust control strategies are strong tools to deal with this dilemma. Although there are different techniques for robust and adaptive control [13], the adaptive approximation control (AAC) is a powerful tool to deal with this topic effectively. The latter technique requires representation of the unknown parameters or the dynamic matrices (inertia matrix, centripetal and Coriolis matrix, gravity vector and so on) in terms of weighting coefficient and basis function matrices. Then, updating adaptive laws for the weighting coefficient matrices are designed based on Lyapunov theory such that the overall system is stable. The reader is referred to [14-19] for more details.

In this work, the AAC is used as a basis for designing the cascade position/torque control loops. The outer loop deals with adaptive position control for the slow link dynamics. Here, a virtual joint torque vector is assumed as an input vector. The virtual velocity error proposed by Slotine and Li [20] is used while the nonlinear terms and unmodeled dynamics are collected in a lumped term to be approximated using the AAC. In a similar manner to the outer position loop, the virtual velocity error and the AAC are used for designing the required control law for the inner torque loop. The overall stability for the nested loops is proved using Lyapunov theory. A two-link manipulator is used as a simulation platform to show the effectiveness of the proposed control structures. In effect, two previous works are similar to our current study which are [3,22]. In [3], regressor-based adaptive control with cascade positiontorque control was used for flexible joint robots. However, it requires calculations of regresor matrix that is highly nonlinear and it is difficult to be applied to high complex robotic systems. In [22] FAT-based adaptive control has been applied to flexible joint robots, however, the control laws include coupled weighting matrices and the problem of noisy signals of the derivative of the desired joint torques were not treated. Our current work can be applied to highly DoF robotic systems and the problems of noisy torque derivative is solved. In essence, the approximation technique is intrinsically decentralized with some modifications, see e.g., our previous work [18]. The contribution of the paper can be summarized as follows:

- Design of a cascade position-torque control based on decoupled adaptive approximation technique.

- It can be efficiently applied to high DoF robotic system due to the inherent parallel computations of the control laws.

- The noisy signals resulted due to the presence of the derivative of the desired joint torque vector is filtered out using a low-pass transfer function. 


\section{DYNAMIC MODELING}

In this section, the dynamic modeling of a multi-link manipulator with flexible joints are described, see Fig. 1(a). Figure 1(b) shows an example of 2-DoF planar robot that will be our target in simulation results. The following assumptions are considered:

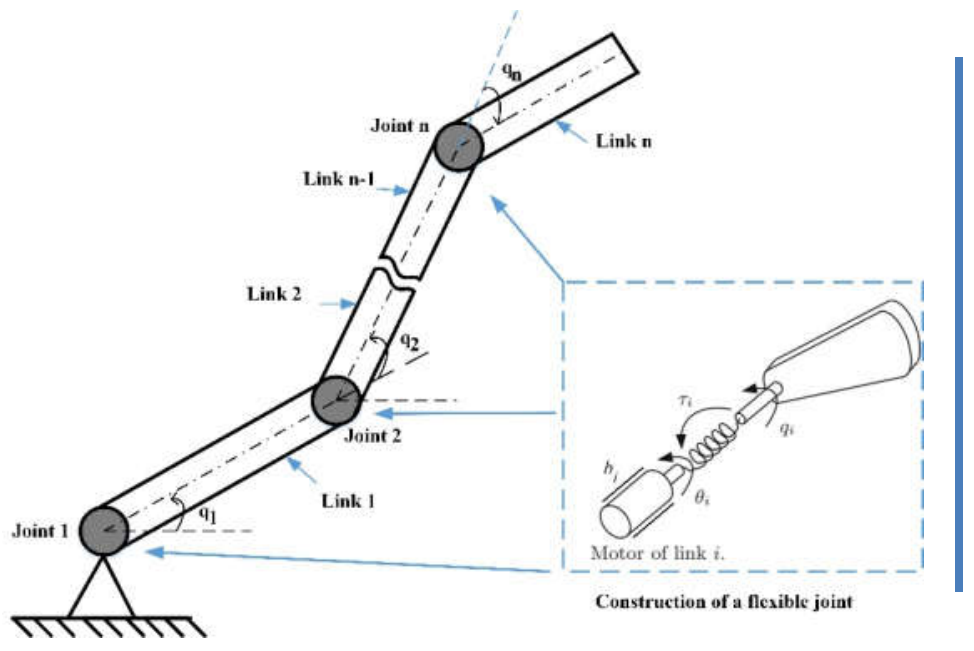

(a)

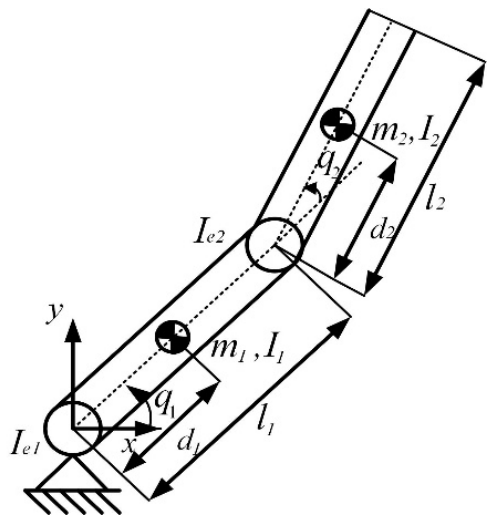

(b)

FIGURE 1. (a) An n-DoF flexible joint robotic manipulator, (b) a two-DoF planar robot. Nomenclatures are defined at the end of the paper.

Assumption 1. The link state variable vector and the joint torque vector resulted from flexible element effect are measureable.

Assumption 2. The diagonal stiffness matrix associated with the flexible element has arbitrary value. No limitations on the value of joint stiffness are imposed.

Thus, according to Euler-Lagrange formulation, the model of flexible-joint robot with $\mathrm{n}$ degrees of freedom (DoFs) can be described as

$$
\begin{gathered}
D(q) \ddot{q}+C(q, \dot{q}) \dot{q}+g(q)=K_{j}(\theta-q) \\
I_{e} \ddot{\theta}+B_{e} \dot{\theta}+K_{j}(\theta-q)=u
\end{gathered}
$$

Where $q \in R^{n \times 1}$ is the angular position of the joints, $D(q) \in R^{n \times n}$ is a symmetric and positive definite inertia matrix, $C(q, \dot{q}) \in R^{n \times n}$ is the centripetal and Coriolis matrix, $g(q) \in R^{n \times 1}$ is the gravity vector, $K_{j} \in R^{n \times n}$ is a diagonal spring stiffness, $\theta \in R^{n \times 1}$ is the angular position of the motor, $I_{e} \in R^{n \times n}$ is a diagonal equivalent inertia matrix of the rotor/gear transmission, $B_{e} \in R^{n \times n}$ is an equivalent diagonal viscous damping matrix, and $u \in R^{n \times 1}$ is an input control vector.

Remark 1. In effect, the motor dynamics should be considered in robots with fast motions and highly varying loads, otherwise it could be neglected. In this work, the actuator dynamics is neglected while equivalent inertia is used instead. See our previous work on control of robots considering actuator dynamics [18].

The dynamic modeling of Eq. (1) has the following properties.

Property 1. The inertia matrix $D(q)$ is uniformly bounded from above and below.

Property 2. Each unknown parameter (or coefficient matrix) in robot modeling can be linearly represented in terms of weighting coefficient and basis function vectors.

The joint torque vector $\tau$ (resulted from the torsional spring) is expressed as

$$
\tau=K_{j}(\theta-q)
$$


Exploiting Eq. (2b), Eq. (1b) can be reformulated as

$$
\theta=K_{j}^{-1} \tau+q
$$

$$
I_{e} K_{j}^{-1} \ddot{\tau}+B_{e} K_{j}^{-1} \dot{\tau}+I_{e} \ddot{q}+B_{e} \dot{q}+\tau=u
$$

Equation (1a) can be modified as follows

$$
\ddot{q}=D(q)^{-1}(\tau-C(q, \dot{q}) \dot{q}-g(q))
$$

Thus substituting Eq. (2d) into Eq. (2c), Eq. (1) can be re-written as

with

$$
\begin{gathered}
D(q) \ddot{q}+C(q, \dot{q}) \dot{q}+g(q)=\tau \\
I_{j} \ddot{\tau}+B_{j} \dot{\tau}+\eta_{j}=u
\end{gathered}
$$

where $I_{n}$ is an $n \times n$ identity matrix.

Remark 2. It should be noted that Eq. (1) can be formulated in different ways such that different controllers are designed. However, this work focuses on a cascade control structure. Accordingly, Eq. (3a) shows that the state variable vector (output) is $q$ with joint torque $\tau$ as a virtual input control. On the other hand, in Eq. (3b) the state variable vector is joint torque vector $\tau$ with the $u$ working as an input voltage control. Consequently, two nested control loops are possible to be established.

Remark 3. The problem associated with dynamics of Eq. (3) is that the number of DoFs is twice of input controls and hence an additional virtual input control is required to stabilize the target dynamic system.

\section{CONTROL STRUCTURE}

As aforementioned, the flexible joint robot is a fourth order dynamic system due to the presence of the flexible element between the load and the actuator drive system. This flexibility creates additional angular position on the actuator side that is different from the joint (load) position. The proposed controller consists of two control loops under assumptions 1 and 2 such that the major (outer loop) attempts to track the desired references of the robot position with a virtual joint torque vector being tracked by the minor (inner) loop; the details are as follows.

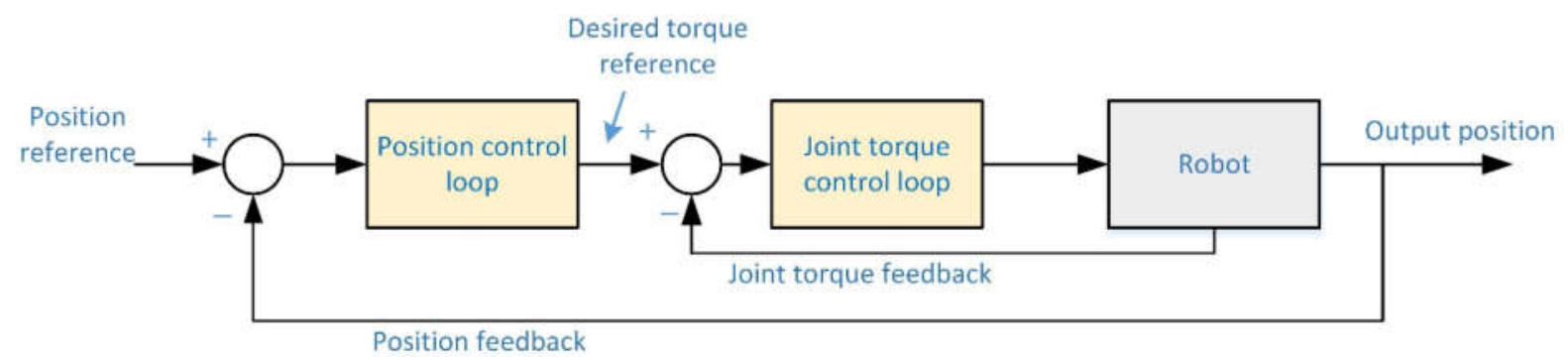

Figure 2. The proposed control structure

\section{The outer position loop}

The desired joint torque vector can be designed as [14-19]

$$
\tau_{d}=\widehat{D} \dot{v}_{p}+\hat{C} v_{p}+\hat{g}+K_{D p} s_{p}+\kappa \operatorname{sgn}\left(s_{p}\right)
$$

where the symbol (^) refers to estimated value, $K_{D p} \in R^{n \times n}$ is a position feedback gain matrix with a positive definite property, $\kappa \in R^{n \times n_{1}}$ is a diagonal matrix associated with the last robust term and

$$
\begin{gathered}
s_{p}=v_{p}-\dot{q} \\
v_{p}=\dot{q}_{d}+\gamma_{p}\left(q_{d}-q\right)
\end{gathered}
$$


with $q_{d} \in R^{n}$ being the desired joint trajectory, $s_{p} \in R^{n}$ is the velocity error, $v_{p} \in R^{n}$ is a reference velocity, and $\gamma_{p}=$ $\operatorname{diag}\left(1 / \lambda_{p 1}, \ldots, 1 / \lambda_{p n}\right)$, where $\lambda_{p i}$ is the sliding time constant.

Using Eq. (5a), Eq. (3a) can be expressed as

$$
\tau=D\left(\dot{v}_{p}-\dot{s}_{p}\right)+\mathrm{C}\left(v_{p}-s_{p}\right)+g
$$

Using the idea of approximation technique, Eq. (6a) becomes

with

$$
\tau=W_{p}^{T} F_{p}-D \dot{s}_{p}-C s_{p}+\epsilon_{p}
$$

$$
D \dot{v}_{p}+C v_{p}+g=W_{p}^{T} F_{p}+\epsilon_{p}
$$

where $W_{p} \in R^{n \beta \times n}$ represents the weighting coefficient matrix, $F_{p} \in R^{n \beta \times n}$ is the corresponding basis-function matrix, and $\epsilon_{\mathrm{p}} \in R^{n}$ refers to the approximation (modeling) error.

Thus, the control law of Eq. (4) can be reformulated as

with

$$
\tau_{d}=\widehat{W}_{p}^{T} F_{p}+K_{D p} s_{p}
$$

$$
\widehat{W}_{p}^{T} F_{p}=\widehat{D} \dot{v}_{p}+\hat{C} v_{p}+\hat{g}
$$

Subtracting Eq. (7) from Eq. (6a) leads to the following closed-loop dynamics

$$
D \dot{s}_{p}+C s_{p}+K_{D p} s_{p}+\kappa \operatorname{sgn}\left(s_{p}\right)=\widetilde{W}_{p}^{T} F_{p}+\epsilon_{p}+e_{t}
$$

Equation (8) is stable as along as $\widetilde{W}_{p} \rightarrow 0, \epsilon_{p} \rightarrow 0$ and $e_{t} \rightarrow 0$, where $e_{t}=\tau-\tau_{d}$.

\section{The inner joint torque loop}

In this inner (minor loop), the input control is the applied input control vector (u) while the output state variable is the joint torque vector $(\tau)$ associated with flexible element. Therefore, the following control law can be designed

$$
u=\hat{I}_{j} \dot{v}_{j}+\widehat{B}_{j} v_{j}+\hat{\eta}_{j}+K_{D j} s_{j}
$$

where $K_{D j} \in R^{n \times n}$ is a symmetrical positive definite matrix, and

$$
\begin{gathered}
s_{j}=v_{j}-\dot{\tau} \\
v_{j}=\dot{\tau}_{d}+\gamma_{j}\left(\tau_{d}-\tau\right)
\end{gathered}
$$

with

$s_{j} \in R^{n}$ is the joint torque error, $v_{j} \in R^{n}$ is a reference joint torque derivative defined in Eq. (9c), $\gamma_{j}=$ $\operatorname{diag}\left(1 / \lambda_{j 1}, \ldots, 1 / \lambda_{j n}\right) \in R^{n \times n}$, where $\lambda_{j i}$ is the sliding time constant related to the inner torque loop. Using the concept of global approximation technique, Eq. (9a) can be re-expressed as

where Eq. (3b) can be reformulated as follows.

$$
u=\widehat{W}_{j}^{T} F_{j}+K_{D j} s_{j}
$$

$$
I_{j} \ddot{\tau}+B_{j} \dot{\tau}+\eta=W_{j}^{T} F_{j}+\epsilon_{j}
$$

with $\epsilon_{j} \in R^{n}$ referring to the approximation error associated with the inner torque dynamics. Whereas, the following closed-loop dynamics is produced by subtracting Eq. (10a) from Eq. (10b).

Equation (10c) is stable if $\widetilde{W}_{j} \rightarrow 0$ and $\epsilon_{j} \rightarrow 0$.

$$
I_{j} \dot{s}_{j}+B_{j} s_{j}+K_{D j} s_{j}=\widetilde{W}_{j}^{T} F_{j}+\epsilon_{j}
$$

Remark 4: The control law described in Eq. (10a) requires calculation of the first derivative of the desired joint torque resulted from the outer position loop. In [3], the authors proved that the control law is bounded since it will be function to the desired joint references up to the third derivatives, the output joint displacement and its derivative, and the joint torque measurement assuming all the kinematic variables (displacements, velocities and accelerations) are bounded. However, in this paper, we will see that the requirement of presence of derivative of desired joint in Eq. (10a) makes the dynamic response of the input control jumping abruptly at the beginning of the transient region response. Therefore, Albu-Schäffer et al. [23] have avoided using this term in their control law. In this work, an approximation for the derivative is proposed as follows.

$$
\dot{\tau}_{d i}=\tau_{d i}\left(\frac{s}{\sigma_{i} s+1}\right), \mathrm{i}=1,2,3, \ldots, \mathrm{n}
$$


where $\mathrm{s}$ is Laplace variable and $\sigma_{i}$ is a time constant for the low-pass transfer function of (11). This time constant should be smaller than the time constant of the system dynamics.

Theorem 1. The dynamic modeling described in Eq. (3) with the control law, and closed-loop dynamics introduced in Eqs. (7), (10a), (8) and (10c) respectively is stable according to Lyapunov theory provided that:

$$
\begin{gathered}
\dot{\hat{W}}_{p}=-Q_{p}^{-1} F_{p} s_{p}^{T} \\
\dot{\hat{W}}_{j}=-Q_{j}^{-1} F_{j} s_{j}^{T} \\
\gamma_{j}^{T} K_{D j} \gamma_{j}>\frac{1}{4} K_{D p}^{-1}, K_{D j} \geq \Gamma_{j}^{-1}, \text { and } B_{j} \geq 0 .
\end{gathered}
$$

where $Q_{(.)}^{-1} \in R^{n \beta \times n \beta}$ is an adaptation diagonal matrix that is important in estimation process, $\Gamma_{p}$ and $\Gamma_{j}$ are both positive definite matrices such that $\Gamma_{j}=\Gamma_{j}^{T}>0$, and [21]

$$
\begin{gathered}
\Gamma_{j}^{T} \epsilon_{j} \Gamma_{j} \leq \bar{\epsilon}_{j} \\
s_{j}^{T} \epsilon_{j} \leq s_{j}^{T} \Gamma_{j}^{-1} s_{j}+\epsilon_{j}^{T} \Gamma_{j} \epsilon_{j} \leq s_{j}^{T} \Gamma_{j}^{-1} s_{j}+\bar{\epsilon}_{j}
\end{gathered}
$$

where $\bar{\epsilon}_{j}$ is a lower bound for and $\epsilon_{j}$.

Proof:

To prove the validity of Theorem 1, let us consider the following non-negative Lyapunov function

$$
V=\frac{1}{2} s_{p}^{T} D s_{p}+\frac{1}{2} s_{j}^{T} I_{j} s_{j}+\frac{1}{2} \operatorname{tr}\left(\widetilde{W}_{p}^{T} Q_{p} \widetilde{W}_{p}+\widetilde{W}_{j}^{T} Q_{j} \widetilde{W}_{j}\right)+e_{j}^{T} \gamma_{j}^{T} K_{D j} e_{j}
$$

Taking the time derivative of the above equation to get

$$
\dot{V}=s_{p}^{T} D \dot{s}_{p}+\frac{1}{2} s_{p}^{T} \dot{D} s_{p}+s_{j}^{T} I_{j} \dot{s}_{j}+\operatorname{tr}\left(\widetilde{W}_{p}^{T} Q_{p} \dot{\widehat{W}}_{p}+\widetilde{W}_{j}^{T} Q_{j} \dot{\widehat{W}}_{j}\right)+2 e_{j}^{T} \gamma_{j}^{T} K_{D j} \dot{e}_{j}
$$

Substituting Eqs. (8) and (11) in Eq. (16) to get

$$
\begin{aligned}
& \dot{V}= s_{p}^{T}\left(\widetilde{W}_{p}^{T} F_{p}+\epsilon_{p}+e_{j}-C(q, \dot{q}) s_{p}-K_{D p} s_{p}\right)+\frac{1}{2} s_{p}^{T} \dot{D} s_{p}+s_{j}^{T}\left(\widetilde{W}_{j}^{T} F_{j}+\epsilon_{j}-B_{j} s_{j}-K_{D j} s_{j}\right)+2 e_{j}^{T} \gamma_{j}^{T} K_{D j} \dot{e}_{j} \\
&+\operatorname{tr}\left(\widetilde{W}_{p}^{T} Q_{p} \dot{\widehat{W}}_{p}+\widetilde{W}_{j}^{T} Q_{j} \dot{\widehat{W}}_{j}\right) \\
&=s_{p}^{T}\left(\widetilde{W}_{p}^{T} F_{p}+\epsilon_{p}+e_{j}-C(q, \dot{q}) s_{p}-K_{D p} s_{p}-\kappa \operatorname{sgn}\left(s_{p}\right)\right)+\frac{1}{2} s_{p}^{T} \dot{D} s_{p}+s_{j}^{T}\left(\widetilde{W}_{j}^{T} F_{j}+\epsilon_{j}-B_{j} s_{j}-\dot{\mathrm{e}}_{j}^{T} K_{D j} \dot{\mathrm{e}}_{j}\right)- \\
& e_{j}^{T} \gamma_{j}^{T} K_{D j} \gamma_{j} e_{j}+\operatorname{tr}\left(\widetilde{W}_{p}^{T} Q_{p} \dot{\widehat{W}}_{p}+\widetilde{W}_{j}^{T} Q_{j} \dot{\widehat{W}}_{j}\right)
\end{aligned}
$$

Using the property of skew matrix of $(\dot{D}-2 C)$ with some manipulations to obtain

$$
\begin{aligned}
& \dot{V}=-s_{p}^{T} K_{D p} s_{p}+s_{p}^{T} e_{j}-e_{j}^{T} \gamma_{j}^{T} K_{D j} \gamma_{j} e_{j}-\kappa \operatorname{sgn}\left(s_{p}\right)+s_{p}^{T} \epsilon_{p}-s_{j}^{T} K_{D j} s_{j}-s_{j}^{T} B_{j} s_{j}+s_{j}^{T} \epsilon_{j}+\operatorname{tr}\left\{\widetilde { W } _ { p } ^ { T } \left(F_{p} s_{p}^{T}+\right.\right. \\
& \left.\left.Q_{p} \dot{\hat{W}}_{p}\right)+\widetilde{W}_{j}^{T}\left(F_{j} s_{j}^{T}+Q_{j} \dot{\widehat{W}}_{j}\right)\right\} \\
& \quad \dot{V}=-\left[\begin{array}{ll}
s_{p}^{T} & e_{j}^{T}
\end{array}\right] R\left[\begin{array}{l}
s_{p} \\
e_{j}
\end{array}\right]+s_{p}^{T} \epsilon_{p}-\kappa \operatorname{sgn}\left(s_{p}\right)-s_{j}^{T} K_{D j} s_{j}-s_{j}^{T} B_{j} s_{j}+s_{j}^{T} \epsilon_{j}+\operatorname{tr}\left\{\widetilde{W}_{p}^{T}\left(F_{p} s_{p}^{T}+Q_{p} \dot{\hat{W}}_{p}\right)+\right. \\
& \left.\widetilde{W}_{j}^{T}\left(F_{j} s_{j}^{T}+Q_{j} \dot{\hat{W}}_{j}\right)\right\}
\end{aligned}
$$

with

$R=\left[\begin{array}{cc}K_{D p} & -\frac{1}{2} I_{n} \\ -\frac{1}{2} I_{n} & \gamma_{j}^{T} K_{D j} \gamma_{j}\end{array}\right]$

Since the matrix $\mathrm{R}$ is a symmetric, the first inequality of $\mathrm{Eq}(13)$ is satisfied according to [3]. By substituting Eqs. $(12 \mathrm{a}, \mathrm{b})$ and substituting Eqs. (14a,b) we can get

$$
\dot{V}=-\left[\begin{array}{ll}
s_{p}^{T} & e_{j}^{T}
\end{array}\right] R\left[\begin{array}{l}
S_{p} \\
e_{j}
\end{array}\right]+s_{p}^{T} \epsilon_{p}-\sum_{i} \kappa_{i}\left|s_{p_{i}}\right|+-s_{j}^{T} B_{j} s_{j}+-s_{j}^{T}\left(K_{D j}-\Gamma_{j}^{-1}\right) s_{j}+\bar{\epsilon}_{j}
$$

To ensure stability, it is required that $\kappa_{i} \geq \delta_{i}+s_{p_{i}}$ and $K_{D j} \geq \Gamma_{j}^{-1}$ such that $\dot{V}$ is lower bounded guaranteeing the asymptotic system stability when $\mathrm{t} \rightarrow \infty$ according to Barbalat's lemma [20], [22].

Remark 5. In effect, the overall control structure including control laws and the corresponding update laws (Eqs. (7), (10a), and (12)) are fully decoupled with diagonal parameter and gain matrices. Therefore, it is easy to be applied to high DoF robotic manipulators . 


\section{RESULTS AND DISCUSSIONS}

In this section, simulation experiments are performed using MATLAB/SIMULINK software on a 2-DoF planar flexible-joint robotic manipulator (see Fig. 1(b)). The physical parameters of the investigated robot are selected as follows: $\quad l_{1}=l_{2}=0.8[\mathrm{~m}], m_{1}=m_{2}=0.75[\mathrm{~kg}], d_{1}=d_{2}=0.4[\mathrm{~m}], I_{1}=I_{2}=0.03\left[\mathrm{~kg} \cdot \mathrm{m}^{2}\right], I_{e 1}=I_{e 2}=$ $\left[0.024 \mathrm{~kg} \cdot \mathrm{m}^{2}\right], b_{1}=b_{2}=0.01[\mathrm{Nms} / \mathrm{rad}], k_{1}=k_{2}=100[\mathrm{Nm} / \mathrm{rad}]$. It is important to note that the stiffness of the flexible joint is considered constant (not changeable stiffness) and the source of flexibility is developed due to the harmonic drive mechanism. For detailed derivation of modeling of a 2-DoF planar robot with flexible joints, see e.g. $[1,3,22]$. To verify the effectiveness of the proposed controller. The following assumptions are imposed for all simulation experiments:

- The modeling (approximation) error is neglected.

- The number of terms of basis functions $(\beta)$ used for simulation experiments is equal to 11. It is well-known in the field of numerical analysis that increasing the number of terms of approximation polynomials $\beta$ could lead to better accuracy, selecting $\beta=11$ is enough for our experiments.

- Chebyshev polynomials are used for approximation purposes.

During all investigated experiments, the task of the robot is commanded to move from rest to constant desired angles $\left(q_{d 1}=60^{\circ}, q_{d 2}=90^{\circ}\right)$. To regulate the motion of the target robot considering joint flexibility, parameters uncertainty and unmodeled dynamics, a control structure including two nested loops are established, recall Sect. 3. The control parameters and adaptation gains used are introduced in Table 1 considering three cases. In these experiments, investigation of the effect of sliding mode time constant (see Eq. (5b)) on the response speed and stability are presented. According to Fig. 3, selection of a large time constant parameter related to the position loop $\left(\lambda_{p 1}=\right.$ $\lambda_{p 2}=1[s]$ ) leads to slow response; however, decreasing $\lambda_{p i}$ to some smaller values (e.g., $\lambda_{p 1}=\lambda_{p 2}=1 / 70[s]$ ) could cause some oscillations in transient response. On the other hand, the time constant parameter related to joint torque loop $\left(\lambda_{j i}\right)$ should be smaller than $\lambda_{p i}$, otherwise undesired performance can be obtained. This behavior occurs since the inner torque loop should be faster than the outer position loop. Among the three experiments, experiment 2 shows better behavior with well fast response. Figure 4 shows the input control vector under the conditions of experiment 2. As mention previously, due to the presence of derivative of desired joint torque $\left(\dot{\tau}_{d}\right)$ in the control laws described in Eq. (10a), there is an abrupt jump in the control inputs at the beginning of the dynamic response. Using the approximation proposed in Eq. (11), the noise effects are reduced. On the other hand, high values are required for $\left(K_{D p}\right)$ associated with outer position loop while lower value is required for the inner torque loop $\left(K_{D j}\right)$, a detailed comparison for the effect of these parameters is cancelled due to limited space of the paper. It should be noted that for all above experiments the control structure does not need a precise prediction (estimation) for the physical parameters. In effect, sufficient definite orthogonal basis terms are enough to get a feasible control performance [14-19, 22]. On the other hand, the cascade control can improve the stability the system and give some perception for the robot by integration of joint torque with the control structure. However, some constraints on control parameters could be imposed such that the dynamics of the inner loop maybe faster than the outer loop and this could limit the system bandwidth.

Table 1. Control parameters used in simulation experiments

\begin{tabular}{cc}
\hline Case No. & Gain values \\
\hline 1 & $\gamma_{p}=I_{2}, K_{D p}=100 I_{2}, Q_{p}^{-1}=300 I_{2}$, \\
& $\gamma_{j}=70 I_{2}, K_{D j}=20 I_{2}, Q_{j}^{-1}=300 I_{2}$. \\
2 & $\gamma_{p}=20 I_{2}, K_{D p}=100 I_{2}, Q_{p}^{-1}=300 I_{2}$, \\
& $\gamma_{j}=70 I_{2}, K_{D j}=20 I_{2}, Q_{j}^{-1}=300 I_{2}$. \\
3 & $\gamma_{p}=70 I_{2}, K_{D p}=100 I_{2}, Q_{p}^{-1}=300 I_{2}$, \\
& $\gamma_{j}=70 I_{2}, K_{D j}=20 I_{2}, Q_{j}^{-1}=300 I_{2}$. \\
\hline
\end{tabular}



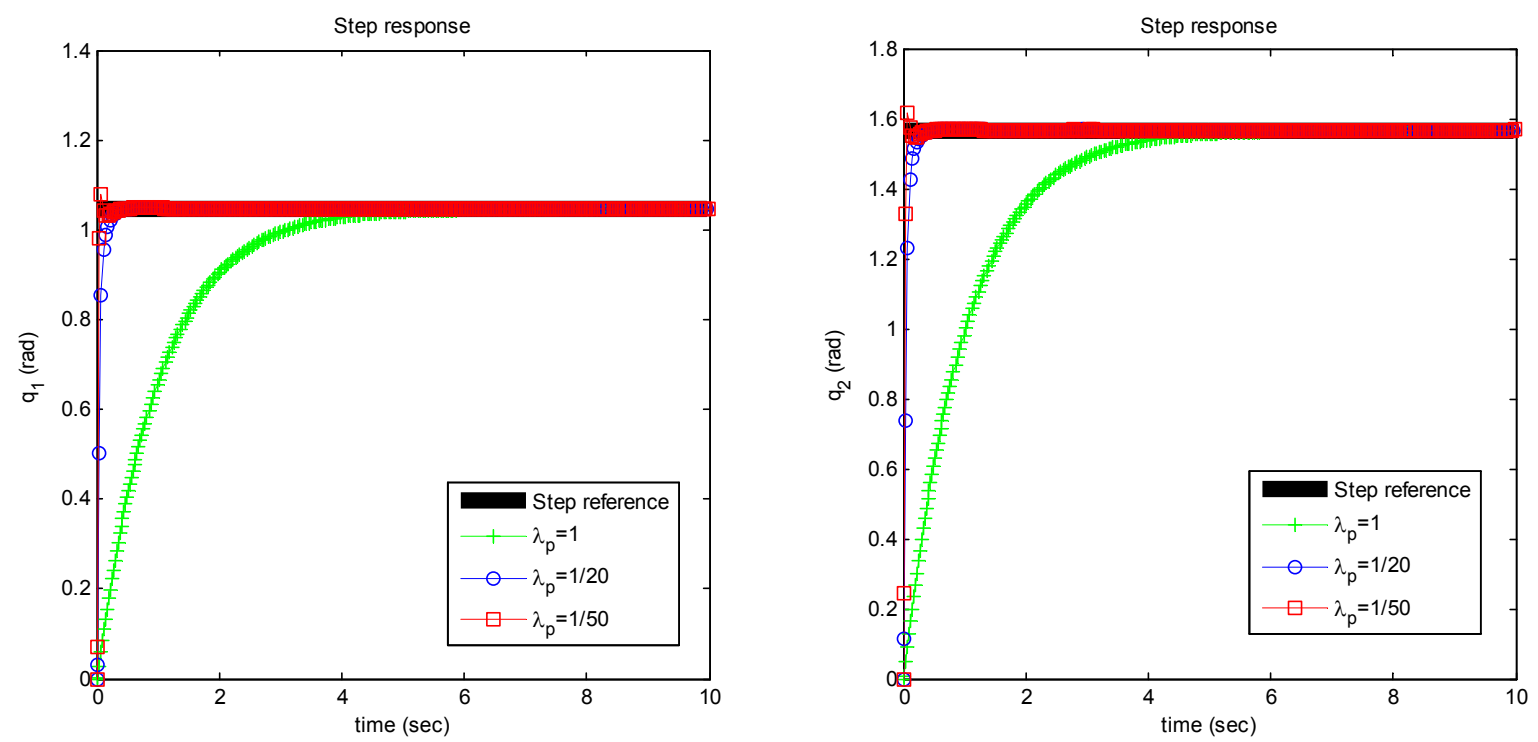

Figure 3. Step position response showing the effect of the sliding mode time constant on response speed.
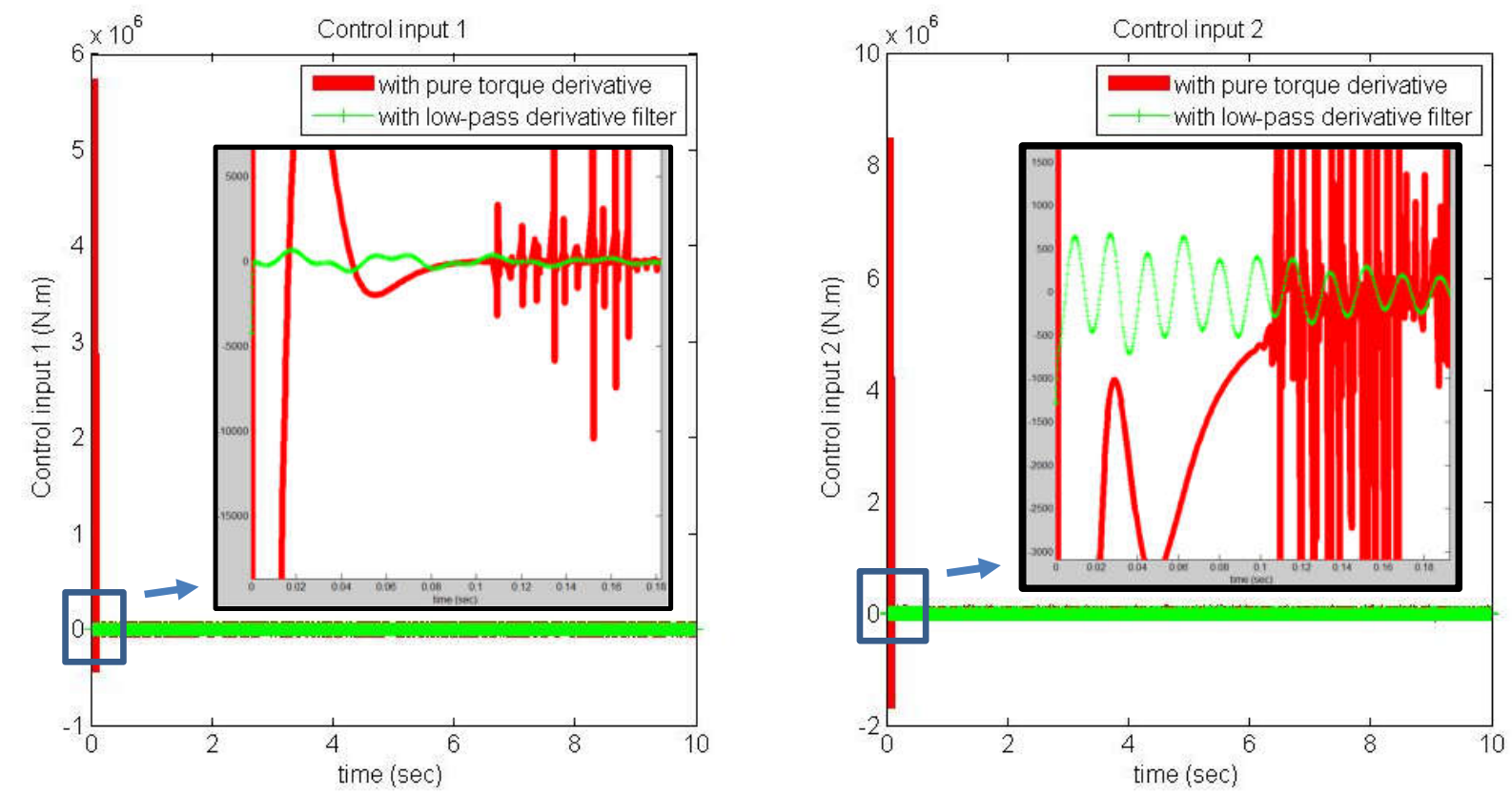

Figure 4. Control inputs with corresponding enlarged views.

\section{CONCLUSIONS}

This paper proposes a cascade position/torque control approach based on adaptive approximation technique. Two nested loops are designed with ensured stability based on Lyapunov theory. Two important points are recognized in the current proposed controller: 1) it is a model free control working well in the presence of model uncertainty, and 2) It is easily to be implemented in decentralized way such that it is qualified for complex robotic systems with high number of DoFs. Despite of the strength of the proposed control structure, the following issues are noted:

- A fully decentralized torque control can be designed to consider the damping effect with flexible elements.

- Design of a generalized controller that deals with flexible components with variable stiffness and damping. 
- Considering more complex robotic systems are important such as humanoid robots.

- Tuning of the control parameters such that the nested loops have ensured stability.

- Experiment work is require in near future to verify the results practically.

\section{LIST OF SYMBOLS AND NOMENCLATURES}

$D(q) \quad$ : a symmetric and positive definite inertia matrix, $\in R^{n \times n}$.

$C(q, \dot{q}) \quad:$ a centripetal and Coriolis matrix, $\in R^{n \times n}$.

$g(q) \quad:$ a gravity vector, $\in R^{n \times 1}$.

$q \quad$ : the angular position of the joints, $\in R^{n \times 1}$.

$K_{j} \quad$ : a diagonal spring stiffness, $\in R^{n \times n}$

$\theta \quad$ : the angular position of the motors, $\in R^{n \times 1}$.

$I_{e} \quad: \quad$ a diagonal equivalent inertia matrix of the rotor/gear transmission, $\in R^{n \times n}$.

$B_{e} \quad$ : an equivalent diagonal viscous damping matrix, $\in R^{n \times n}$.

$u \quad$ : the control input vector, $\in R^{n \times 1}$.

$\tau \quad:$ the joint torque vector, $\in R^{n \times 1}$.

$I_{n} \quad:$ an identity matrix, $\in R^{n \times n}$.

(.) $\quad:$ refers to estimation.

$q_{d} \quad$ : the desired joint trajectory, $\in R^{n \times 1}$.

$s_{(\cdot)} \quad$ : the velocity error, $\in R^{n \times 1}$.

$v_{(.)} \quad:$ a reference velocity, $\in R^{n \times 1}$.

$\lambda_{(.)} \quad$ : a sliding mode time constant.

$\gamma_{(.)} \quad$ : a diagonal constant gain matrix function of sliding mode time constants, $\in R^{n \times n}$.

$\epsilon_{(.)} \quad$ : the modelling (approximation) error, $\in R^{n \times 1}$.

$\widetilde{(.)} \quad:$ the difference between the estimated and actual values.

$\sigma_{i} \quad:$ : a time constant associated with low-pass filter.

$K_{D(.)} \quad$ : a positive definite feedback gain matrix, $\in R^{n \times n}$.

$Q_{(.)}^{-1} \quad:$ an adaptation diagonal matrix, $\in R^{n \beta \times n \beta}$.

$\beta \quad$ : the number of terms of the basis functions.

$\Gamma_{(.)} \quad$ : a constant positive definite diagonal matrix, $\in R^{n \times n}$.

$V \quad:$ Lyapunov function.

$l_{(.)} \quad:$ the link length, $(\mathrm{m})$.

$m_{(\cdot)} \quad$ : the link mass, $(\mathrm{kg})$.

$d_{(\cdot)} \quad$ : the distance of center of mass of a link, (m).

$b_{(.)} \quad$ : the viscous damping coefficient of a joint, $\left(\frac{\mathrm{Nms}}{\mathrm{rad}}\right)$.

\section{DECLARATIONS}

The authors declare no competing interests.

\section{REFERENCES}

1. M. Spong, S. Hutchinson and M. Vidyasagar, Robot modeling and control, John Wiley, 2nd Edition, 2020.

2. N. Kashiri, N.G. Tsagarakis, M. Van Damme, B. Vanderborght, D.G. Caldwell, Proxy-based sliding mode control of compliant joint manipulators. In: Filipe J., Gusikhin O., Madani K., Sasiadek J. (eds) Informatics in Control, Automation and Robotics. Lecture Notes in Electrical Engineering, vol 370. Springer, Cham. 2016. https://doi.org/10.1007/978-3-319-26453-0_14

3. T. Lin, Adaptive and robust control of flexible joint robots with joint torque feedback, PhD Dissertation, Department of Electrical and Computer Engineering, University of Toronto, 1998. 
4. Gen-Liang Xiong, Hai-Chu Chen, Jing-Xin Shi and Fa-Yun Liang, Joint torque control of flexible joint robots based on sliding mode technique. International Journal of Advanced Robotic Systems, 2019, https://doi.org/10.1177/1729881419846712.

5. R.M. Berger and H.A. ElMaraghy, Feedback linearization control of flexible joint robots. Rob Comput Integr Manuf 1992; 9(3): 239-246.

6. H. Dallali, J. Lee, N.G.Tsagarakis, D.G.Caldwell, Experimental study on linear state feedback control of humanoid robots with flexible joints. IFAC-Papers Online,Volume 48, Issue 19, 2015, Pages 130-135, ISSN 2405-8963, https://doi.org/10.1016/j.ifacol.2015.12.022

7. Z.H. Jiang and K. Shinohara, Workspace trajectory tracking control of flexible joint robots based on backstepping method. In: 2016 IEEE region 10 conference (TENCON), Singapore, Singapore, 22-25, 2016.

8. Kim J and Croft EA, Full-state tracking control for flexible joint robots with singular perturbation techniques. IEEE Trans Control Syst. Technol. 2017; 99: 1-13.

9. R. Reyes-Ba'ez and A.J. van der Schaft, Virtual differential passivity based control for tracking of flexible-joints robots. In: Workshop on Lagrangian and Hamiltonian methods in nonlinear control, 1-4 May 2018, pp. 1-8, IFAC.

10. A. Izadbakhsh and M. Masoumi, FAT-based robust adaptive control of flexible-joint robots: singular perturbation approach. In: 2017 IEEE international conference on industrial technology (ICIT), Toronto, Ontario, Canada, 22-25, March 2017, pp. 22-25. IEEE.

11. M.J. Kim and W.K. Chung, Disturbance-observer-based PD control of flexible joint robots for asymptotic convergence. IEEE Trans Robot 2015; 31(6): 1508-1516.

12. Z.J. Li, H.B. Wu, M.J. Yang, M.H. Wang and J.H. We. A Position and Torque Switching Control Method for Robot Collision Safety. Int. J. Autom. Comput. 15, 156-168 (2018). https://doi.org/10.1007/s11633-017-11049

13. Jay A. Farrell, Marios M. Polycarpou, Adaptive Approximation Based Control: Unifying Neural, Fuzzy and Traditional Adaptive Approximation Approaches, John Wiley, 2006.

14. Hayder F. N. Al-Shuka, B. Corves and Wen-Hong Zhu, Function approximation technique-based adaptive virtual decomposition control for a serial-chain manipulator. Robotica, Volume 32, Issue 3, May 2014, pp. 375 - 399. DOI: https://doi.org/10.1017/S0263574713000775

15. H.F.N. Al-Shuka, On local approximation-based adaptive control with applications to robotic manipulators and biped robots. Int. J. Dynam. Control 6, 339-353 (2018). https://doi.org/10.1007/s40435-016-0302-6

16. H.F.N. Al-Shuka and R. Song, "Hybrid Regressor and Approximation-Based Adaptive Control of Robotic Manipulators with Contact-Free Motion," 2018 2nd IEEE Advanced Information Management, Communicates, Electronic and Automation Control Conference (IMCEC), 2018, pp. 325-329, doi: 10.1109/IMCEC.2018.8469628.

17. Hayder F.N. Al-Shuka, R. Song, Adaptive Hybrid Regressor and Approximation Control of Robotic Manipulators in Constrained Space. International Journal of Mechanical and Mechatronics Engineering, 17(03), 11-21, 2017.

18. H.F.N. Al-Shuka, R. Song, Decentralized adaptive partitioned approximation control of high degrees-of-freedom robotic manipulators considering three actuator control modes. Int. J. Dynam. Control 7, 744-757 (2019). https://doi.org/10.1007/s40435-018-0482-3

19. Hayder F.N. Al-Shuka, FAT-Based Adaptive Backstepping Control of an Electromechanical System with an Unknown Input Coefficient, FME Transactions, 49 (1), 113-121, 2021.

20. J.-J.E. Slotine and W. Li, Applied nonlinear control, Prentice Hall, Pearson Education, 1991.

21. Wen Yu, Xiaoou Li, and George W. Irwin: Stable Anti-Swing Control for an Overhead Crane with Velocity Estimation and Fuzzy Compensation. R. Lowen and A. Verschoren (eds.), Foundations of Generic Optimization, Volume 2: Applications of Fuzzy Control, Genetic Algorithms and Neural Networks, 223-240, 2008.

22. An-Chyau Huang and Ming-Chih Chien, Adaptive control of robot manipulators: a unified regressor-free approach. World Scientific Publishing Co. Pte. Ltd, 2010.

23. Alin Albu-Schäffer, Christian Ott and Gerd Hirzinger. A Unified Passivity-based Control Framework for Position, Torque and Impedance Control of Flexible Joint Robots. The International Journal of Robotics Research 2007; 26; 23. DOI: 10.1177/0278364907073776 